\title{
Three novel microRNAs based on microRNA signatures for gastric mucosa- associated lymphoid tissue lymphoma
}

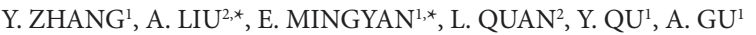 \\ ${ }^{1}$ Department of Radiation Oncology, Harbin Medical University Cancer Hospital, Harbin, Heilongjiang Province 150040, China; ${ }^{2}$ Department \\ of Hematology, Harbin Medical University Cancer Hospital, Harbin, Heilongjiang Province 150040, China \\ *Correspondence: liuaich56@163.com; mingyanmy88@sina.com
}

Received February 8, 2017 / Accepted July 12, 2017

\begin{abstract}
This study aimed to identify novel microRNAs (miRNAs) that play crucial regulatory roles in the pathogenesis of mucosaassociated lymphoid tissue (MALT) lymphoma by retrieving and analyzing the miRNA expression profile GSE23877. Differentially expressed miRNAs between gastric MALT lymphoma samples and human tonsil tissue samples as well as their target genes were identified. The transcriptional regulatory relationships between miRNAs and target genes were analyzed, and the regulatory network between them was constructed. Target genes annotated as transcription factors (TFs) were screened, and a miRNA-target gene regulatory network was established. Moreover, the expression levels of miRNAs and target genes as well as the correlation between them were verified. In total, 53 upregulated and 25 downregulated miRNAs were obtained, for which 35 and 25 experimentally validated miRNA-target interactions, respectively, were screened. Some miRNAs were significantly enriched in certain pathways; for example, miR-320a was enriched in systemic lupus erythematosus and ribosome, miR-622 in the p53 signaling pathway and chronic myeloid leukemia, and miR-429 in cancer-related pathways. In addition, upregulated miRNAs, including miR-320a, miR-940, and miR-622, and downregulated miRNAs, including miR-331-3p and miR-429, were hub nodes in the miRNA-target gene regulatory network, and the TF MYC was a co-target of miR-320a, miR-622, and miR-429. The expression trends of miR-320a and miR-429 as well as of some of their target genes were consistent with those in the results of microarray analysis. In conclusion, miR-320a, miR-622, and miR-429 are possibly novel miRNAs participating in the pathomechanism of gastric MALT lymphoma.
\end{abstract}

Key words: Mucosa-associated lymphoid tissue lymphoma, microRNA, microRNA expression profiling

Mucosa-associated lymphoid tissue (MALT) lymphoma, first described in 1983, generally occurs in MALT because of chronic antigenic stimulation [1]. It is a common type of lymphoma in extranodal sites and affects several organs, predominantly the gastrointestinal tract [2]. Chronic infection with Helicobacter pylori is a major risk factor for MALT lymphoma [3].

MicroRNAs (miRNAs) are crucial post-transcriptional elements that modulate expression of genes involved in the development, cell differentiation and proliferation, and immune responses in cancer initiation and progression [4]. One previous study has suggested that miR-155-enhanced autophagy plays an important role in the immune response against $H$. pylori and is a potential target for the clinical management of $H$. pylori-related diseases, such as MALT lymphoma [5]. Because the etiology of MALT lymphoma is largely unknown, identifying dysregulated miRNAs and investigating their biological significance would be very important for the progress in developing treatments for MALT lymphoma.

Saito et al. have found that miR-142-5p and miR-155 are overexpressed in gastric MALT lymphoma [6]. Vanessa et al. have also identified that miR-34a overexpression has strong anti-proliferative effects on lymphoma cells and that its target, FoxP1, is involved in malignant transformation [7]. Some upregulated miRNAs, such as miR-150/155, as well as downregulated miRNAs, such as miR-184, miR-200a/b/c, and miR-205, have also been found in conjunctival MALT lymphoma and thus might be important in the pathogenesis and progression of this disease [8]. In addition, by analyzing the miRNA expression profile GSE23877, Craig et al. have revealed that miR-203 is strongly downregulated and participates in the transformation from chronic gastritis to MALT lymphoma [9]. However, more information on this profile 
still needs to be analyzed to identify more miRNAs involved in the pathomechanism of MALT lymphoma. Thus, in this study, we aimed to identify novel miRNAs that play crucial regulatory roles in the pathogenesis of this disease.

\section{Materials and methods}

Gene expression profile data. The miRNA expression profile GSE23877 [9] was retrieved from the NCBI's Gene Expression Omnibus database. This dataset has been generated by Craig et al. from five freshly frozen human gastric MALT lymphoma samples and four human tonsil tissue samples [9]. The platform of the profile is GPL10701 (Agilent-021827 Unrestricted Human 15.7K v3.0 miRNA Microarray).

Microarray data preprocessing. The original microarray data file (.txt) was preprocessed by AgiMicroRna (version: $2.18 .0)[10,11]$ in R. The widely used preprocessing strategy Robust multiarray average for Affymetrix microarray data from the "affy" package [12] was applied. The preprocessing procedure included the background correction of intensities, normalization of intensities, and $\log 2$ transformation of probe intensities.

Differential miRNA expression analysis. The R package limma (version: 3.24.15) [3] was then used to identify differentially expressed miRNAs between gastric MALT lymphoma samples and control samples. Moderated t-statistics were obtained using Empirical Bayes methods, and the p-value was adjusted using the Benjamini and Hochberg method. The thresholds set for identifying differentially expressed miRNAs in the analysis were adjusted p-values of $<0.01$ and $\mid \log _{2}$ fold change, $\mathrm{FC} \mid$ of $>2$.

Target gene analysis. The publicly available database MiRWalk 2.0 (http://zmf.umm.uni-heidelberg.de/apps/zmf/ mirwalk2/) is the largest source of information on predicted and experimentally validated miRNA-target interactions [13]. To guarantee the accuracy of the subsequent analysis, only experimentally validated miRNA-target interactions were selected.

Pathway enrichment analysis of target genes. Kyoto Encyclopedia of Genes and Genomes (KEGG, http://www. genome.ad.jp/kegg/) provides information for reconstructing metabolic and non-metabolic pathways [14]. ClusterProfiler (version: 2.2.7) [15] in R was used to identify KEGG pathways enriched by target genes and differentially expressed miRNAs. Pathways with p-values of $<0.05$ were considered significantly enriched by genes and miRNAs.

Transcriptional regulatory analysis between miRNAs and target genes. Target genes and differentially expressed miRNAs that were significantly enriched in pathways were obtained, and the regulatory network between them was constructed using Cytoscape (version: 3.2.1) [16] software. In addition, lymphoma-related genes in the network were identified using the Comparative Toxicogenomics Database (CTD, http://ctdbase.org/) [17], a public resource that provides information about interactions between chemicals in the environment and genes and their relationship to diseases. Information on lymphoma-related markers/therapeutic targets was downloaded from CTD on May 25, 2016.

Relationship between miRNAs and transcription factors (TFs). Target genes annotated as TFs are more likely to be regulated by miRNAs [18]. Therefore, exploring the regulatory relationships between miRNAs and TFs would be helpful to understand the roles of miRNAs in the pathomechanism of the disease. Therefore, the Animal Transcription Factor DataBase 2.0 (http://bioinfo.life.hust. edu.cn/AnimalTFDB/download_index?tr=Homo_sapiens) [19], a comprehensive database for the expression, prediction, and functional annotation of TFs, was used in this study to analyze TFs involved in the miRNA-target gene regulatory network.

Validation of gene and miRNA expression by RT-qPCR. To validate differentially expressed miRNAs identified by gene expression profiling technologies and the expression levels of target genes regulated by differentially expressed miRNAs, the human B-lymphoblast cell line HMy2.CIR and four human B-cell lymphoma lines (Raji, Daudi, CA46, and Farage) were used. CA46 and Farage were obtained from the China Center for Type Culture Collection (Wuhan, China), and other cell lines were obtained from Shanghai Institutes for Biological Sciences, Chinese Academy of Sciences (Shanghai, China). HMy2.CIR cells were grown in $90 \%$ IMDM medium, and Raji, Daudi, CA46, and Farage were grown in 90\% RPMI-1640 medium supplemented with $10 \%$ FBS in a humidified incubator at $37^{\circ} \mathrm{C}$ with $5 \% \mathrm{CO}_{2}$.

The total RNA of each cell was extracted using Takara RNAisoPlus (9109; Takara, Japan). Total RNA was 3'-end labeled using Escherichia coli poly(A) polymerase, followed by reverse transcription reactions using miRNA First-Strand cDNA Synthesis Kit (EY001; Shanghai Iyunbio Information Technology Limited Company, Shanghai, China). mRNA was reverse-transcribed using PrimeScript RT Master Mix (RR036A, Takara). A real-time quantitative RT-PCR analysis was performed to detect miRNA and mRNA levels by SYBRGreen PCR amplification (1504490; Applied Biosystems, Foster City, CA, USA). All primers are shown in Supplementary Table 1. GAPDH was used as a loading control.

Validation of miRNA-target interaction. The 3'-UTR sequence (1208 bp) of Homo sapiens PLCG1 (NCBI Reference Sequence: NM_002660.2) was cloned into the multicloning site of the pmirGLO luciferase reporter vector. $293 \mathrm{~T}$ cells plated in a 24 -well plate at $70 \%$ confluence were co-transfected with pmirGLO-PLCG1 and miR-429 mimic or mimic NC. 293T cells co-transfected with pmirGLO and miR-429 mimic/miR-429 were used as controls. Co-transfection of plasmids and siRNAs was performed with Lipofectamine 2000, in accordance with the manufacturer's protocol (11668-027; Invitrogen, Carlsbad, CA, USA). After co-transfection for $24 \mathrm{~h}$, luciferase expression in transfected cells was detected using the Dual-Luciferase Reporter Assay 
kit, in accordance with the manufacturer's protocol (RG027; Beyotime Institute of Biotechnology, China).

Statistical analysis. SPSS22.0 and GraphPad Prism 5.0 software (GraphPad Software, San Diego, CA, USA) were used for statistical analysis. t-test and non-parametric test procedures were used. A ${ }^{*}$ p-value of $<0.05$ was considered statistically significant, and ${ }^{* *}$ p-value of $<0.01$ was considered to indicate a very significant difference.

\section{Results}

Data normalization and differentially expressed miRNAs. The heat map shown in Figure 1 displays the expression profiles of all differentially expressed miRNAs. In total, 53 upregulated and 25 downregulated miRNAs were obtained.

Target genes of differentially expressed miRNAs. Experimentally validated miRNA-target interactions were obtained for 35 of the 53 upregulated miRNAs, and miRNAs-target interactions of six of the 25 downregulated miRNAs were screened. In total, 1466 genes were targeted by overexpressed
miR-149-3p, 660 were targeted by overexpressed miR-665, and only four were targeted by miR-1469. miR-455-3p with low expression targeted 552 genes, whereas the number of target genes of miR-342-5p was the lowest $(n=111)$ (Table 1$)$.

KEGG pathway enriched by differentially expressed miRNAs. In total, 14 upregulated miRNAs and two downregulated miRNAs were significantly enriched in pathways (Figure 2A and 2B). The six pathways most significantly enriched by the upregulated miRNAs were systemic lupus erythematosus (miR-760 and miR-320a), p53 signaling pathway (miR-622), ribosome (miR-320a), circadian rhythm - mammal (miR-1290 and hsa-miR-1246), chronic myeloid leukemia (miR-622 and miR-422a), and neurotrophin signaling pathway (miR-622 and miR-422a). Meanwhile, hsa-miR-429 with low expression was mainly involved in cancer-related pathways and signaling pathways, including the MAPK signaling pathway, the Wnt signaling pathway, and the T-cell receptor signaling pathway.

miRNA-target gene network. The miRNA-target gene regulatory network comprised 206 target genes and 39 miRNAs (Figure 3). In total, 33 upregulated miRNAs,

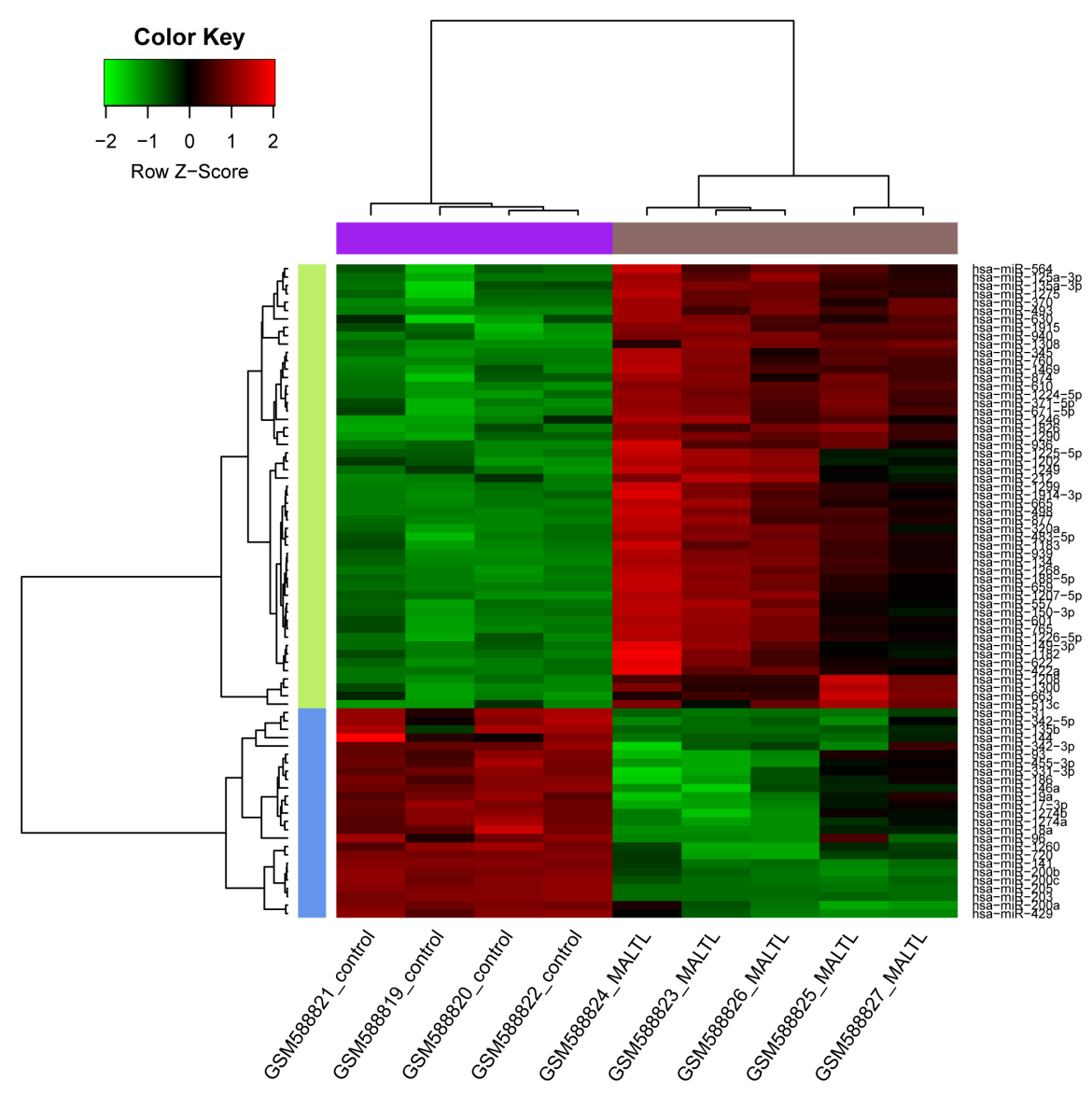

Figure 1. Heat map summarizing microRNA expression. Colors represent relative microRNA expression as indicated in the color key. Red and green indicate upregulation and downregulation, respectively, relative to the overall mean for each microRNA. 

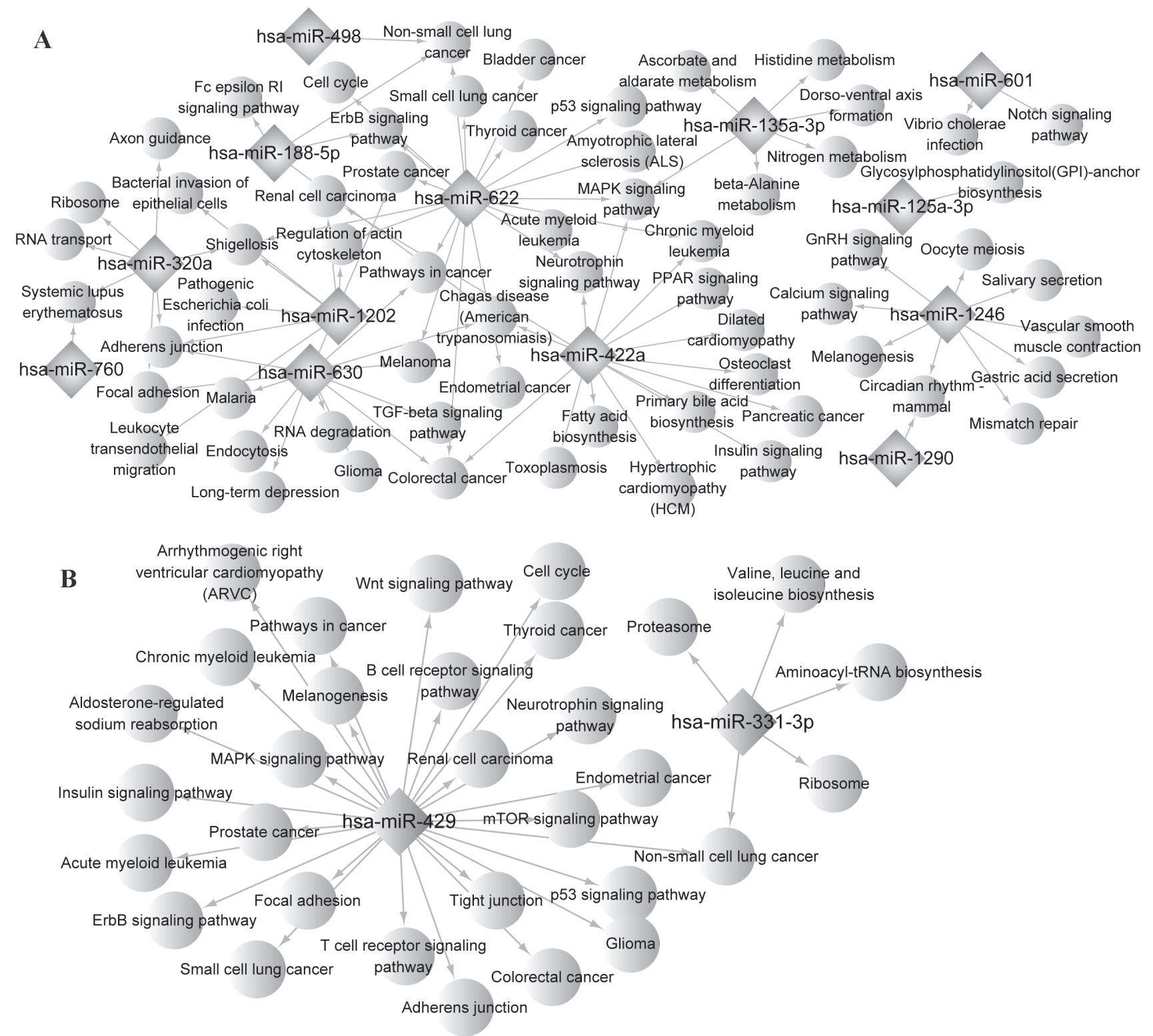

Figure 2. Kyoto Encyclopedia of Genes and Genomes pathways enriched by upregulated microRNAs (A) and downregulated microRNAs (B). The diamonds represent microRNAs, and the circles represent pathways.

including the hub nodes miR-760, miR-320a, miR-940, and miR-622, and six downregulated miRNAs, including the hub nodes miR-331-3p and miR-429 were found. In addition, 13 experimentally validated lymphoma-related targets were found (Table 2). For example, MAPK1 and PTEN were miR-320a targets; TP53, MYC, and KRAS were miR-622 targets; and BCL2, MALT1, CDKN1B, and PLCG1 were miR-429 targets.

The relationship between miRNAs and TFs. The miRNA-TF regulatory network is shown in Figure 4. In total, 18 TFs were regulated by 21 differentially expressed miRNAs. The downregulated miRNAs, including miR-429 and miR-455-3p, and 14 upregulated miRNAs, including miR-622 and miR-149-3p, were hub nodes in the network. MYC was the co-target of miR-320a, miR-622, miR-429, miR-940, and miR-125a-3p.

The expression levels of miRNAs detected by RT-qPCR. The results of RT-qPCR (Figure 5) showed that the upregulated miR-320a was highly expressed in four human B-lymphoblast cell lines. The expression of experimentally validated lymphoma-related targets of miR-320a was detected. MAPK1 was downregulated in Raji, Daudi, and Farage, but was upregulated in CA46, and PTEN was downregulated in four B-lymphoma cell lines. The downreg- 
Table 1. Target genes of differentially expressed microRNAs

\begin{tabular}{lclc}
\hline miRNA & Target count & $\begin{array}{l}\text { miRNA } \\
\text { Upregulated }\end{array}$ & Target count \\
\hline hsa-miR-149-3p & 1466 & hsa-miR-630 & 83 \\
hsa-miR-665 & 660 & hsa-miR-1182 & 82 \\
hsa-miR-940 & 643 & hsa-miR-1290 & 82 \\
hsa-miR-320a & 571 & hsa-miR-1226-5p & 78 \\
hsa-miR-765 & 355 & hsa-miR-1202 & 64 \\
hsa-miR-125a-3p & 292 & hsa-miR-1208 & 64 \\
hsa-miR-498 & 282 & hsa-miR-1246 & 52 \\
hsa-miR-1207-5p & 223 & hsa-miR-1225-5p & 49 \\
hsa-miR-760 & 216 & hsa-miR-150-3p & 44 \\
hsa-miR-557 & 207 & hsa-miR-135a-3p & 38 \\
hsa-miR-671-5p & 164 & hsa-miR-422a & 34 \\
hsa-miR-1275 & 155 & hsa-miR-601 & 18 \\
hsa-miR-483-5p & 145 & hsa-miR-1469 & 4 \\
hsa-miR-188-5p & 136 & & 163 \\
hsa-miR-622 & 118 & & 513 \\
hsa-miR-1914-3p & 117 & Downregulated & \\
hsa-miR-1183 & 108 & hsa-miR-455-3p & 552 \\
hsa-miR-610 & 95 & hsa-miR-331-3p & 41 \\
hsa-miR-564 & 90 & hsa-miR-342-3p & 351 \\
hsa-miR-1224-5p & 84 & hsa-miR-17-3p & 163 \\
hsa-miR-1299 & 84 & hsa-miR-429 & \\
hsa-miR-936 & 84 & hsa-miR-342-5p & \\
\hline
\end{tabular}

Table 2. Differentially expressed microRNAs (miRNAs) and the experimentally validated lymphoma-related targets.

\begin{tabular}{ll}
\hline $\begin{array}{l}\text { Marker/therapeutic } \\
\text { gene (regulated) }\end{array}$ & miRNA (regulator) \\
\hline PDPK1 & hsa-miR-1183, hsa-miR-331-3p, hsa-miR-429 \\
TP53 & hsa-miR-1207-5p, hsa-miR-622, hsa-miR-149-3p \\
ATP6AP1 & hsa-miR-1226-5p, hsa-miR-601, hsa-miR-455-3p \\
CREBBP & hsa-miR-601 \\
KRAS & hsa-miR-671-5p, hsa-miR-622, hsa-miR-429 \\
PLCG1 & hsa-miR-765, hsa-miR-188-5p, hsa-miR-1914-3p, \\
hsa-miR-429 & hsa-miR-940, hsa-miR-125a-3p, hsa-miR-622, \\
CYC & hsa-miR-320a, hsa-miR-429, hsa-miR-455-3p \\
CDKN1B & hsa-miR-149-3p, hsa-miR-429 \\
PTEN & hsa-miR-320a, hsa-miR-429 \\
MAPK1 & hsa-miR-320a, hsa-miR-498 \\
BCL2 & hsa-miR-630, hsa-miR-429 \\
EP300 & hsa-miR-429 \\
MALT1 & hsa-miR-429 \\
\hline
\end{tabular}

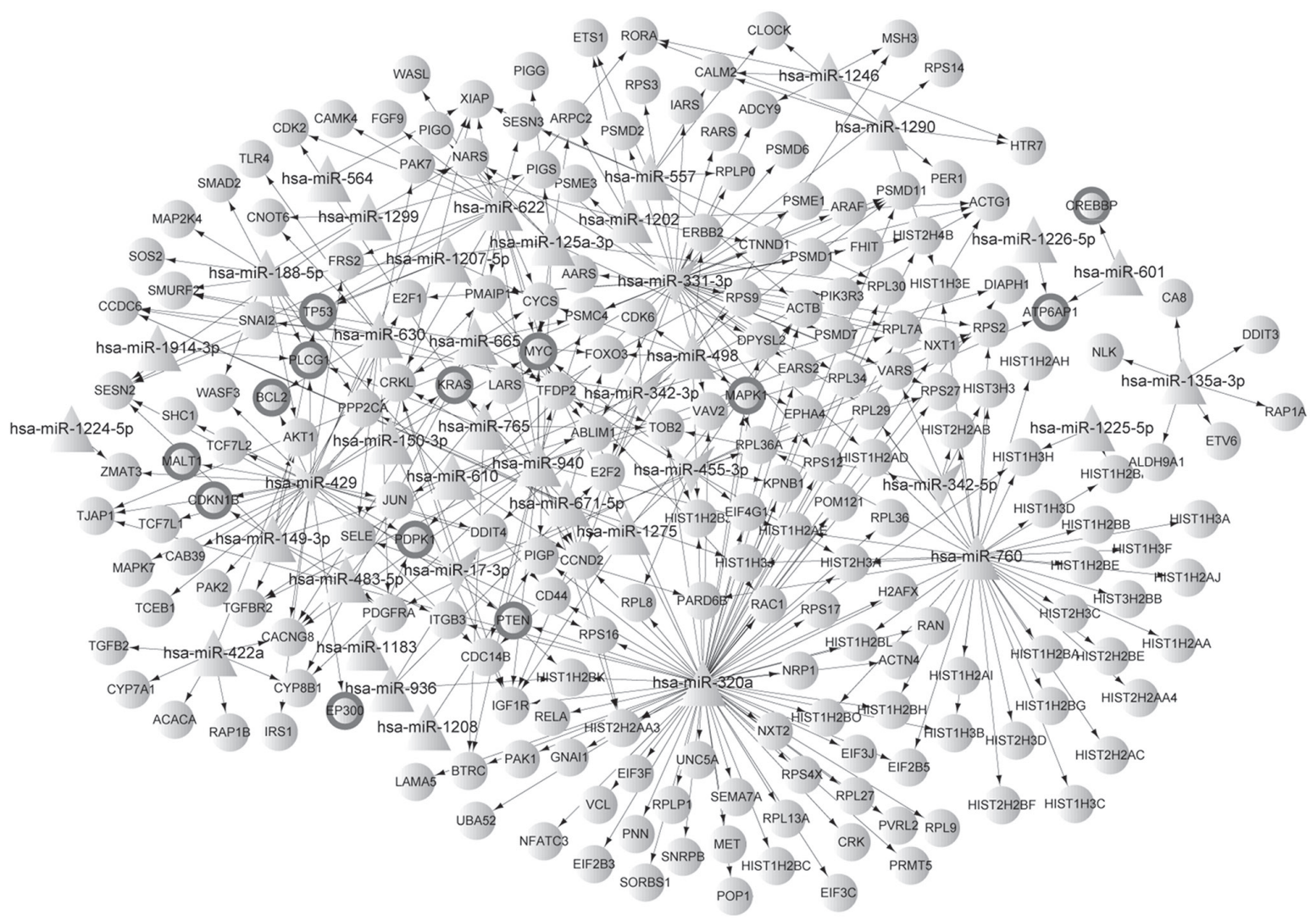

Figure 3. MicroRNA-target gene regulatory network. The upper triangles and lower gray triangles represent upregulated and downregulated microRNAs, respectively. The circles represent target genes, and those with black edges represent experimentally validated lymphoma-related targets. 
Figure 4. MicroRNA-transcription factor (TF) regulatory network. The upper triangles and lower gray triangles represent upregulated and downregulated microRNAs, respectively. The circles represent TFs.

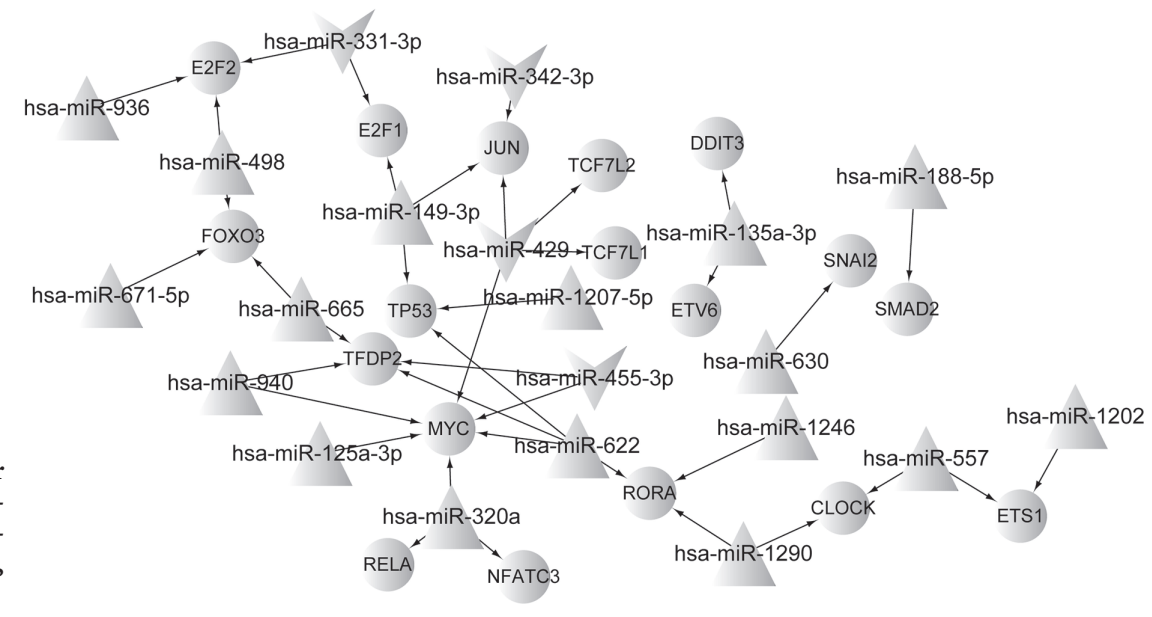

miR-320a

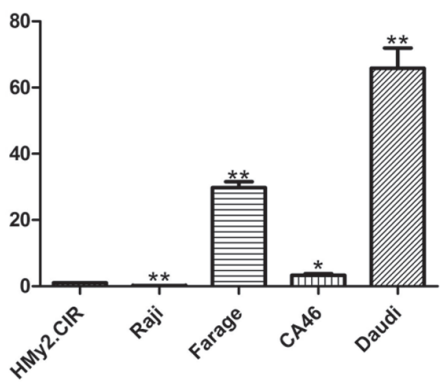

$\operatorname{miR}-429$

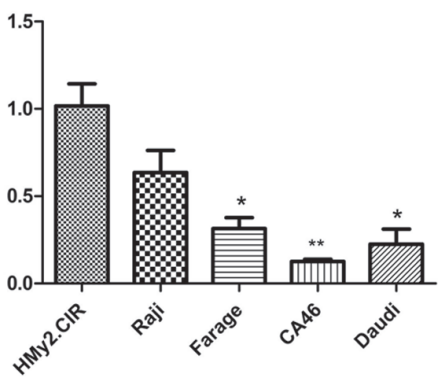

CDKN1B

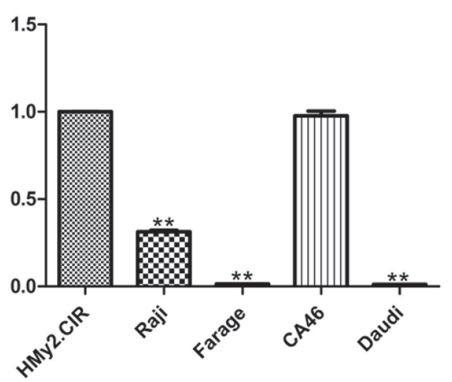

MAPK1

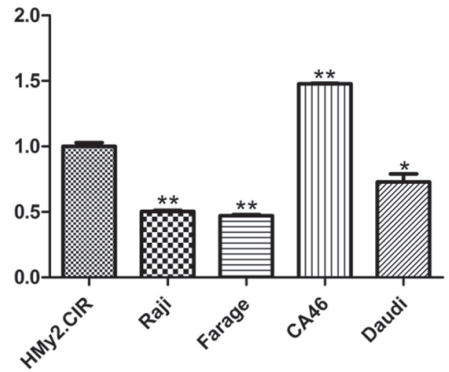

BCL2

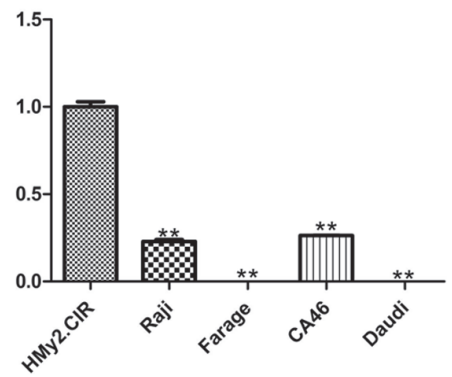

PLCG1

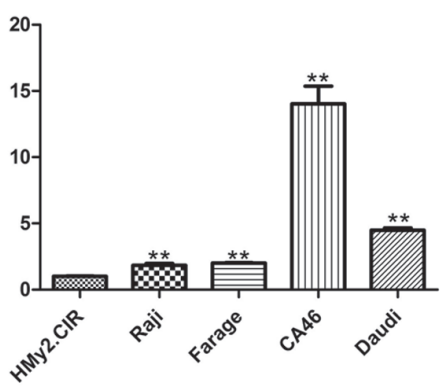

PTEN

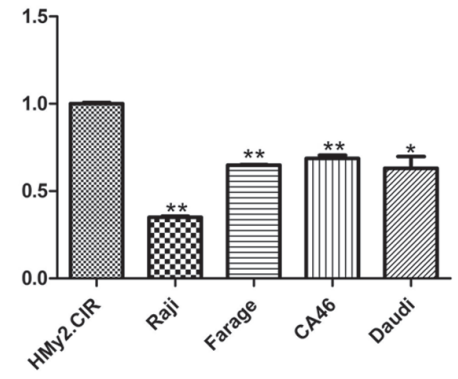

MALT1

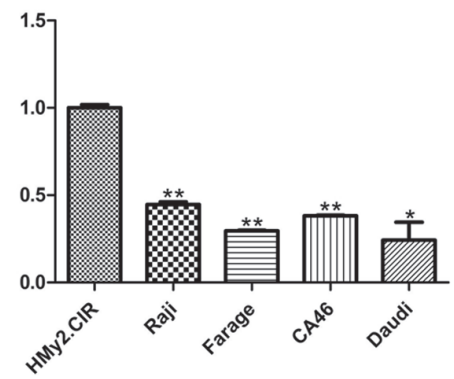

miR-622

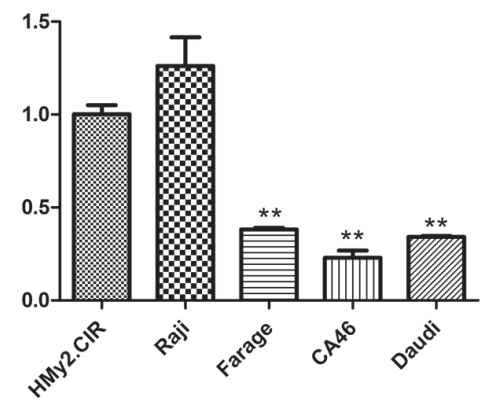

Figure 5. Relative expression levels of hsa-miR-320a hsa-miR-429 and hsa-miR-622 as well as target genes of hsa-miR-320a and hsa-miR-429 in the human B-lymphoblast cell line HMy2.CIR and four human B-cell lymphoma lines, namely Raji, Daudi, CA46, and Farage. MAPK1 and PTEN were hsa-miR-320a targets. BCL2, MALT1, CDKN1B, and PLCG1 were hsa-miR-429 targets. ${ }^{*}, \mathrm{p}<0.05$ compared with that of HMy2.CIR; ${ }^{* *}, \mathrm{p}<0.01$ compared with that of HMy2.CIR. 
ulated miR-429 was expressed at a low level in Daudi, CA46, and Farage, and the same trend of expression was observed in Raji, with no significant difference. miR-429 targets, including BCL2, MALT1, and CDKN1B, were downregulated, whereas PLCG1 was upregulated in these four cell lines. miR-622 was significantly downregulated in Farage, CA46, and Daudi, and upregulated in Raji, but the difference was not significant.

MiRNA-target interaction. The interaction between PLCG1 and miR-429 was validated. The results in Figure 6 show that compared with the other three cells, those co-transfected with pmirGLO-PLCG1 and miR-429 had significantly decreased relative luciferase activity. This indicates the target interaction between PLCG1 and miR-429.

\section{Discussion}

In this study, we aimed to identify novel miRNAs participating in the pathomechanism of gastric MALT lymphoma based on the miRNA expression profile. The results revealed 53 upregulated and 25 downregulated miRNAs. Subsequently, miRNA target genes were identified and their functions, as well as those of miRNAs, were explored. Some miRNAs, including upregulated miR-320a, miR-940, and miR-622, and downregulated miR-331-3p and miR-429 were enriched in the miRNA-target gene network and the miRNA-TF regulatory network.

The upregulated miRNAs miR-320a and miR-622 were enriched in the miRNA-target gene network and were found to be involved in many pathways. Previous studies have suggested that miR-320a expressed at a low level suppressed tumor cell proliferation and migration in colon cancer [20] and hepatocellular cancer [21]. In addition, Sheng et al. have found that an inhibitor of miR-320a can effectively reduce Ebola virus-induced cytotoxicity and that the cell adhesion-related genes TFPI and DAG1 as miR-320a targets are associated with the integrity of blood vessels and cell viability [22]. Our results showed that some miR-320a targets were enriched in pathways, including systemic lupus erythematosus, focal adhesion, and shigellosis. Systemic lupus erythematosus is closely related to non-Hodgkin's lymphoma (NHL), and a few cases reports have also revealed its connection with MALT lymphoma [23]. In addition, MAPK1 and PTEN were identified as miR-320a targets. Abnormally expressed PTEN in cancers is related to the function of gastrointestinal MALT [24]. MAPK1 is also involved in cellular proliferation and differentiation in cancers [25]. Interestingly, downregulated miR-622 has been found to promote cellular proliferation and invasion in cancers, e.g., gastric cancer [26], glioma [27], and hepatocellular carcinoma [28]. In this study, miR-622 was found to be mostly enriched in cancer-related pathways. A mutation of KRAS, one of the miR-622 targets, has been reported in cutaneous T-cell lymphoma [29] as well as in other cancers [30]. Moreover, MYC, a TF related to both miR-622 and

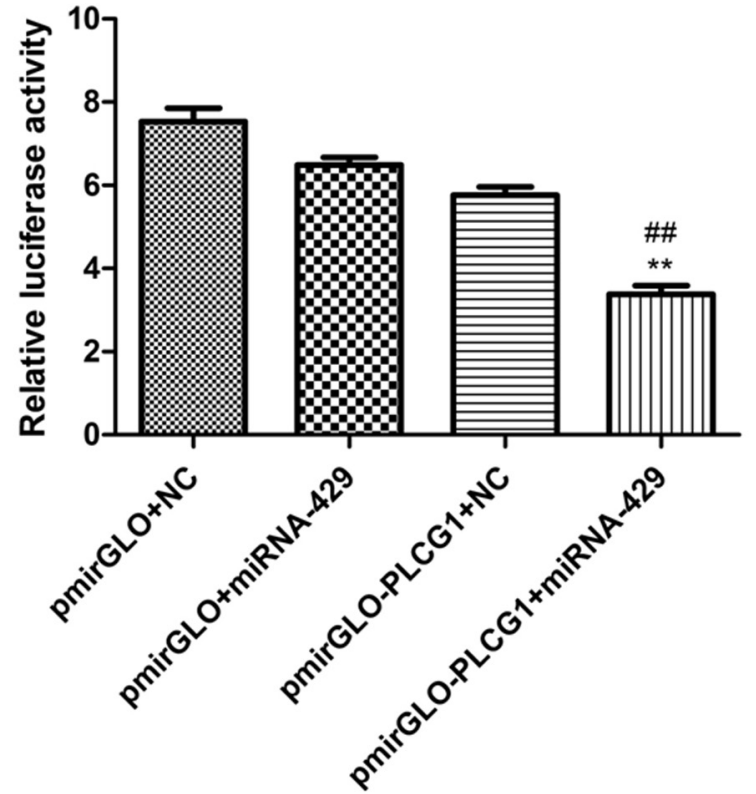

Figure 6. The relative luciferase activity in four cells co-transfected with pmirGLO-PLCG1 and hsa-miR-429 mimic/mimic NC or with pmirGLO and hsa-miR-429 $\mathrm{mimic} / \mathrm{mimic}$ NC. ${ }^{* *} \mathrm{p}<0.01$ compared with that co-transfected with pmirGLO-PLCG1 and hsa-miR-429 mimic NC. $\# \# \mathbf{p}<0.01$ compared with that co-transfected with pmirGLO and hsamiR-429 mimic NC.

miR-320a, was found to be another miR-622 target. MYC has been shown to be associated with lymphomas, including Burkitt lymphoma [31] and aggressive B-cell lymphomas [32]. Therefore, miR-320a and miR-622 possibly play important roles in the progression and development of gastric MALT lymphoma.

miR-429 plays a role in the metastasis of hepatocellular carcinoma via the classic Wnt pathway [33]. It targets c-myc and acts as a recessive cancer gene involved in the pathogenesis of gastric carcinoma [34]. Furthermore, it is downregulated and may function as a tumor suppressor by targeting PLCG1 in breast cancers [35]. Overexpressed miR-429 can induce apoptosis and the suppression of esophageal carcinoma cell invasion [36]. In our study, miR-429 was also found in multiple cancer-related pathways, the MAPK signaling pathway, the T-cell receptor signaling pathway, and the Wnt signaling pathway. In addition, it was shown to mediate the expression of MALT1, BCL2, and PDPK1, among others. The proteins encoded by MALT1 [37], BCL2 [38], and PDPK1 [39] have been found to be associated with B-cell lymphoma of MALT. In addition, miR-429 had a close connection with the TF MYC. As mentioned above, miR-429 could be another important miRNA involved in the pathomechanism of gastric MALT lymphoma.

The RT-qPCR analysis of miR-320a and miR-429 expression confirmed the accuracy and acceptable sensitivity of the microarray results. However, only a few miRNA targets 
displayed a negative correlation with these miRNAs in terms of their expression patterns. This may have occured because the expression of genes is regulated by multiple miRNAs and some other factors [40]. Moreover, the target correlation between PLCG1, showing negative trend in expression toward miR-429, and miR-429 was identified. This further confirmed the accuracy of the bioinformatic prediction.

Bioinformatic analysis can be used to predict possible key genes or pathways associated with a disease. The advantage of such analysis is that it can simply and quickly obtain useful information from large amounts of data. However, bioinformatic analysis has the disadvantage of providing predictive results, and thus may not be reliable. Thus, an experimental verification of bioinformatic results is needed. In the present study, the results of bioinformatic analysis indicated that miR-622 was upregulated in the gastric MALT lymphoma samples compared with that in the control samples. However, the results of RT-qPCR showed that miR-622 was significantly downregulated in Farage, CA46, and Daudi, and upregulated in Raji, but the difference was not significant. Given the predictive nature of bioinformatic analyses as mentioned above, it is not unusual to find inconsistency between the results of bioinformatic analyses and RT-qPCR, which can be due to the different types and sources of samples as well as other factors. Although inconsistency was noted between the results of bioinformatic analysis and RT-qPCR for miR-622, miR-622 was differentially expressed in the gastric MALT lymphoma samples compared with its expression in the control sample.

In addition, Raji, Daudi, CA46, and Farage are four human B-cell lymphoma lines with different origins. The Raji cell line (EBNA-positive) has been derived from a B-lymphocyte of an 11-year-old Nigerian male with Burkitt lymphoma from 1963 [41], which was established by Pulvertaft. In contrast, the Daudi cell line (EBNA-positive) has been from the peripheral blood of a 16-year-old black male with Burkitt lymphoma [42]. Daudi features typical B lymphoblasts and is widely used to study the pathogenesis of leukemia. Moreover, the CA46 cell line (EBNAnegative) has been established from the ascites of a person with Burkitt lymphoma, whereas the Farage cell line has been established from a lymph node biopsy of a patient with NHL. The cells lack both T-cell and myeloid surface markers; express B-cell surface antigens, including CD19, CD20, CD22, and HLA-DR; are positive for C3 receptors and EBNA; and express BCL-2 [43]. However, as the sources of these four cell lines differed, differences were also present in their miRNA and target gene expression.

In conclusion, the findings of this study indicate that miR-320a, miR-622, and miR-429 are likely to be functionally related to each other as they have the same targets, such as MYC. All of these miRNAs may be important in the pathomechanism of gastric MALT lymphoma. However, further experimental validation is needed to confirm our results.

\section{References}

[1] WILLIAMSON R, WELCH CE, MALT RA. Adenocarcinoma and lymphoma of the small intestine. Distribution and etiologic associations. Ann Surg 1983; 197: 172.

[2] RADASZKIEWICZ T, DRAGOSICS B, BAUER P. Gastrointestinal malignant lymphomas of the mucosa-associated lymphoid tissue: factors relevant to prognosis. Gastroenterology 1992; 102: 1628-1638.

[3] PEREIRA M-I, MEDEIROS JA. Role of Helicobacter pylori in gastric mucosa-associated lymphoid tissue lymphomas. World J Gastroenterol 2014; 20: 684-698. https://doi. org/10.3748/wjg.v20.i3.684

[4] BELAIR C, DARFEUILLE F, STAEDEL C. Helicobacter pylori and gastric cancer: possible role of microRNAs in this intimate relationship. Clin Microbiol Infect 2009; 15: 806-812. https://doi.org/10.1111/j.1469-0691.2009.02960.x

[5] WU K, ZHU C, YAO Y, WANG X, SONG J et al. MicroRNA155-enhanced autophagy in human gastric epithelial cell in response to Helicobacter pylori. Saudi J Gastroenterol 2016; 22: 30-36. https://doi.org/10.4103/1319-3767.173756

[6] SAITO Y, SUZUKI H, TSUGAWA H, IMAEDA H, MATSUZAKI $J$ et al. Overexpression of miR-142-5p and miR155 in gastric mucosa-associated lymphoid tissue (MALT) lymphoma resistant to Helicobacter pylori eradication. PLoS One 2012; 7: e47396. https://doi.org/10.1371/journal. pone.0047396

[7] CRAIG VJ, COGLIATTI SB, IMIG J, RENNER C, NEUENSCHWANDER $S$ et al. Myc-mediated repression of microRNA-34a promotes high-grade transformation of B-cell lymphoma by dysregulation of FoxP1. Blood 2011; 117: 6227-6236. https://doi.org/10.1182/blood-2010-10-312231

[8] CAI J, LIU X, CHENG J, LI Y, HUANG X et al. MicroRNA-200 is commonly repressed in conjunctival MALT lymphoma, and targets cyclin E2. Graefes Arch Clin Exp Ophthalmol 2012; 250: 523-531. https://doi.org/10.1007/ s00417-011-1885-4

[9] CRAIG V, COGLIATTI S, REHRAUER H, W NDISCH T, M LLER A. Epigenetic silencing of microRNA-203 dysregulates ABL1 expression and drives Helicobacter-associated gastric lymphomagenesis. Cancer Res 2011; 71: 3616-3624. https:// doi.org/10.1158/0008-5472.CAN-10-3907

[10] COONEN M, THEUNISSEN DH, KLEINJANS JC, JENNEN DG. MagiCMicroRna: a web implementation of AgiMicroRna using shiny. Source Code Biol Med 2015; 10: 4. https://doi.org/10.1186/s13029-015-0035-5

[11] LOPEZ-ROMERO P. Pre-processing and differential expression analysis of Agilent microRNA arrays using the AgiMicroRna Bioconductor library. BMC Genomics 2011; 12: 64. https://doi.org/10.1186/1471-2164-12-64

[12] IRIZARRY RA, HOBBS B, COLLIN F, BEAZER-BARCLAY YD, ANTONELLIS KJ et al. Exploration, normalization, and summaries of high density oligonucleotide array probe level data. Biostatistics 2003; 4: 249-264. https://doi.org/10.1093/ biostatistics/4.2.249

[13] DWEEP H, GRETZ N. miRWalk2. 0: a comprehensive atlas of microRNA-target interactions. Nat Methods 2015; 12: 697-697. https://doi.org/10.1038/nmeth.3485 
[14] KANEHISA M. The KEGG database. pp 91-103. In: Novartis Foundation (Eds.). 'In Silico' Simulation of Biological Processes, John Wiley \& Sons, New York, 2002, p. 270. ISBN 97804708448099

[15] YU G, WANG LG, HAN Y, HE QY. clusterProfiler: an R package for comparing biological themes among gene clusters. OMICS 2012; 16: 284-287. https://doi.org/10.1089/ omi.2011.0118

[16] SHANNON P, MARKIEL A, OZIER O, BALIGA NS, WANG JT et al. Cytoscape: a software environment for integrated models of biomolecular interaction networks. Genome Res 2003; 13: 2498-2504. https://doi.org/10.1101/gr.1239303

[17] DAVIS AP, GRONDIN CJ, LENNON-HOPKINS K, SARACENI-RICHARDS C, SCIAKY D et al. The Comparative Toxicogenomics Database's 10th year anniversary: update 2015. Nucleic Acids Res 2015; 43: D914-D920. https://doi. org/10.1093/nar/gku935

[18] ENRIGHT AJ, JOHN B, GAUL U, TUSCHL T, SANDER C et al. MicroRNA targets in Drosophila. Genome Biol 2003; 5: R1. https://doi.org/10.1186/gb-2003-5-1-r1

[19] ZHANG H-M, LIU T, LIU C-J, SONG S, ZHANG X et al. AnimalTFDB 2.0: a resource for expression, prediction and functional study of animal transcription factors. Nucleic Acids Res 2015; 43: D76-D81. https://doi.org/10.1093/nar/gku887

[20] SUN J-Y, HUANG Y, LI J-P, ZHANG X, WANG L et al. MicroRNA-320a suppresses human colon cancer cell proliferation by directly targeting $\beta$-catenin. Biochem Biophys Res Commun 2012; 420: 787-792. https://doi.org/10.1016/j. bbrc.2012.03.075

[21] YAO J, LIANG L-H, ZHANG Y, DING J, TIAN Q et al. GNAI1 suppresses tumor cell migration and invasion and is post-transcriptionally regulated by $\mathrm{mir}-320 \mathrm{a} / \mathrm{c} / \mathrm{d}$ in hepatocellular carcinoma. Cancer Biol Med 2012; 9: 234-241. https://doi.org/10.7497/j.issn.2095-3941.2012.04.003

[22] SHENG M, ZHONG Y, CHEN Y, DU J, JU X et al. HsamiR-1246, hsa-miR-320a and hsa-miR-196b-5p inhibitors can reduce the cytotoxicity of Ebola virus glycoprotein in vitro. Sci China Life Sci 2014; 57: 959-972. https://doi. org/10.1007/s11427-014-4742-y

[23] GONZLEZN, XICOY B, OLIV A, JOV J, RIBERA JMetal. Systemic lupus erythematosus in a patient with primary MALT lymphoma of the larynx. Ear Nose Throat J 2009; 88: E4-5

[24] HEINDL M, H NDEL N, NGEOW J, KIONKE J, WITTEKIND $\mathrm{C}$ et al. Autoimmunity, intestinal lymphoid hyperplasia, and defects in mucosal B-cell homeostasis in patients with PTEN hamartoma tumor syndrome. Gastroenterology 2012; 142: 1093-1096. https://doi.org/10.1053/j.gastro.2012.01.011

[25] XU J, XIAO-QIANG E, WANG NX, WANG MN, XIE HX et al. BMP7 enhances the effect of BMSCs on extracellular matrix remodeling in a rabbit model of intervertebral disc degeneration. FEBS J 2016; 283: 1689-1700. https://doi. org/10.1111/febs.13695

[26] GUO X-B, JING CQ, LI LP, ZHANG L, SHI YL et al. Downregulation of miR-622 in gastric cancer promotes cellular invasion and tumor metastasis by targeting ING1 gene. World J Gastroenterol 2011; 17: 1895-1902. https://doi.org/10.3748/ wjg.v17.i14.1895
[27] ZHANG R, LUO H, WANG S, CHEN Z, HUA L et al. miR622 suppresses proliferation, invasion and migration by directly targeting activating transcription factor 2 in glioma cells. J Neurooncol 2015; 121: 63-72. https://doi.org/10.1007/ s11060-014-1607-y

[28] SONG WH, FENG XJ, GONG SJ, CHEN JM, WANG SM et al. microRNA-622 acts as a tumor suppressor in hepatocellular carcinoma. Cancer Biol Ther 2015; 16: 1754-1763. https://doi.org/10.1080/15384047.2015.1095402

[29] KIE LING MK, OBERHOLZER PA, MONDAL C, KARPOVA MB, ZIPSER MC et al. High-throughput mutation profiling of CTCL samples reveals KRAS and NRAS mutations sensitizing tumors toward inhibition of the RAS/RAF/MEK signaling cascade. Blood 2011; 117: 2433-2440. https://doi. org/10.1182/blood-2010-09-305128

[30] LIEVRE A, BACHET JB, BOIGE V, CAYRE A, LE CORRE $\mathrm{D}$ et al. KRAS mutations as an independent prognostic factor in patients with advanced colorectal cancer treated with cetuximab. J Clin Oncol 2008; 26: 374-379. https://doi. org/10.1200/JCO.2007.12.5906

[31] VALERA A, EPISTOLIO S, COLOMO L, RIVA A, BALAGUE $\mathrm{O}$ et al. Definition of MYC genetic heteroclonality in diffuse large B-cell lymphoma with 8q24 rearrangement and its impact on protein expression. Mod Pathol 2016; 29: 844-853. https://doi.org/10.1038/modpathol.2016.71

[32] TIAN X, PELTON A, SHAHSAFAEI A, DORFMAN DM. Differential expression of enhancer of zeste homolog 2 (EZH2) protein in small cell and aggressive B-cell nonHodgkin lymphomas and differential regulation of EZH2 expression by $\mathrm{p}$-ERK1/2 and MYC in aggressive B-cell lymphomas. Mod Pathol 2016; 29: 1050-1057. https://doi. org/10.1038/modpathol.2016.114

[33] TANG J, LI L, HUANG W, SUI C, YANG Y et al. MiR-429 increases the metastatic capability of HCC via regulating classic Wnt pathway rather than epithelial-mesenchymal transition. Cancer Lett 2015; 364: 33-43. https://doi.org/10.1016/j. canlet.2015.04.023

[34] SUN T, WANG C, XING J, WU D. miR-429 modulates the expression of c-myc in human gastric carcinoma cells. Eur J Cancer 2011; 47: 2552-2559. https://doi.org/10.1016/j. ejca.2011.05.021

[35] ULHMANN S, ZHANG J, SCHWAGER A, MANNSPERGER H, RIAZALHOSSEINI Y et al. miR-200bc/429 cluster targets PLCgammal and differentially regulates proliferation and EGF-driven invasion than miR-200a/l41 in breast cancer. Oncogene 2010; 29: 4297-4306. https://doi.org/10.1038/ onc.2010.201

[36] WANG Y, LI M, ZANG W, MA Y, WANG N et al. MiR-429 up-regulation induces apoptosis and suppresses invasion by targeting Bcl-2 and SP-1 in esophageal carcinoma. Cell Oncol 2013; 36: 385-394. https://doi.org/10.1007/s13402-0130144-6

[37] AKAGI T, MOTEGI M, TAMURA A, SUZUKI R, HOSOKAWA $Y$ et al. A novel gene, MALT1 at 18q21, is involved in $\mathrm{t}(11 ; 18)(\mathrm{q} 21 ; \mathrm{q} 21)$ found in low-grade B-cell lymphoma of mucosa-associated lymphoid tissue. Oncogene 1999; 18: 5785-5794. 
[38] PAN L, DISS T, CUNNINGHAM D, ISAACSON P. The bcl-2 gene in primary B cell lymphoma of mucosa-associated lymphoid tissue (MALT). Am J Pathol 1989; 135: 7-11.

[39] FANG X, ZHOU X, WANG X. Clinical development of phosphatidylinositol 3-kinase inhibitors for non-Hodgkin lymphoma. Biomarker research 2013; 1: 1. https://doi. org/10.1186/2050-7771-1-1

[40] CHEN K, RAJEWSKY N. The evolution of gene regulation by transcription factors and microRNAs. Nat Rev Genet 2007; 8: 93-103. https://doi.org/10.1038/nrg1990
[41] PULVERTAFT R. A study of malignant tumours in Nigeria by short-term tissue culture. J Clin Pathol 1965; 18: 261-273.

[42] BILLOT K, PARIZOT C, ARROUSS I, MAZIER D, DEBRE $P$ et al. Differential aiolos expression in human hematopoietic subpopulations. Leukemia Res 2010; 34: 289-293. https:// doi.org/10.1016/j.leukres.2009.05.016

[43] BEN-BASSAT H, POLLIACK A, SHLOMAI Z, KOHN G, HADAR R et al. Farage, a novel early B cell lymphoma cell line with trisomy 11. Leuk Lymphoma 1992; 6: 513-521. https://doi.org/10.3109/10428199209053591 